\title{
Routing on a weighted scale-free network
}

\author{
Mao-Bin $\mathrm{Hu}^{1,2},{ }^{*}$ Rui Jiang ${ }^{1}$, Yong-Hong $\mathrm{Wu}^{2},{ }^{\dagger}$ Wen-Xu Wang ${ }^{3}$, and Qing-Song $\mathrm{Wu}^{1}$ \\ ${ }^{1}$ School of Engineering Science, University of Science and Technology of China, Hefei 230026, P.R.China \\ ${ }^{2}$ Department of Mathematics and Statistics, \\ Curtin University of Technology, Perth WA6845, Australia \\ ${ }^{3}$ Department of Electronic Engineering, Arizona State University, Tempe Arizona 85287-5706, USA
}

(Dated: April 1, 2008)

\begin{abstract}
In this paper, the packet traffic flow on weighted scale-free networks is investigated based on the local routing strategy using link weights: $P_{l \rightarrow i}=\frac{w_{l i}^{\alpha}}{\sum_{j} w_{l j}^{\alpha}}$. The capacity of links is controlled by $\max \left(\beta w_{l j}, 1\right)$, and the capacity of nodes is controlled by node strength: $\max \left(\gamma s_{i}, 1\right)$. It is shown by simulations that the traffic dynamics depends strongly on the navigation parameters. These behaviors can be explained by investigating the average number of packets on nodes and delivered through links.
\end{abstract}

Keywords: Weighted scale-free networks; Routing strategy; Traffic capacity

\section{INTRODUCTION}

Ever since the small-world [1] and scale-free properties [2] were identified, complex networks have received much attention from physicists, mainly because a wide range of systems in nature and society could be described by complex networks. Research has been focused on topologies of networks as well as dynamics upon networks, including traffic dynamics of information packets, spreading of rumors and epidemic, cooperation behaviors of evolutionary games, synchronization dynamics, navigation and search ability, and so on [3-9]. Due to the importance of large communication networks such as the Internet and WWW in modern society, many investigations have been focused on ensuring free traffic flow and avoiding traffic congestion on complex networks [10-14].

*Electronic address: humaobin@ustc.edu.cn

${ }^{\dagger}$ Electronic address: y. wu@curtin.edu. au 
Recently, some models have been proposed to mimic the traffic routing on complex networks by introducing packets generating rate $R$ and homogeneously selected sources and destinations of data packets [12-17]. In these models, the capacity of networks is measured by a critical generating rate $R_{c}$. At this critical rate, a continuous phase transition from free flow state to congested state occurs. In the free state, the numbers of created and delivered packets are balanced, leading to a steady state. While in the jammed state, the number of accumulated packets increases with time due to the limited delivering capacity or finite queue length of each node. A variety of studies have been focused on developing better packet routing strategies to enhance traffic flow and to avoid traffic congestion on a growing large communication network. In previous studies, packets are forwarded following the random walking [15, 18], the shortest path [16], the efficient path [17], the nearest-neighbor search strategy [19-21], the next-nearest-neighbor search strategy [22], the local information [19-21] or the integration of local static and dynamic information [23].

In most real cases, a complex topology is often associated with a large heterogeneity in the capacity and intensity of the connections, i.e., most networks are weighted networks. However, the traffic dynamics have been investigated mainly in un-weighted networks. And the previous traffic routing strategies have been focused on how to route the packets by using local or global node information (mainly by using node degree). Moreover, weights have a strong correlation with the network topology [24-29] and the existing weighted features play a significant role in a variety of dynamical processes [31-33]. Therefore, a modeling approach that can capture the effects of weighted characteristics on traffic dynamics is need. In this paper, a study of traffic dynamics in a weighted scale-free network is carried out with a conceptual traffic model in which packets are guided based on local link information with a single tunable parameter $\alpha$. To maximize the capacity of the networks which can be measured by the critical packet generating rate $R_{c}$, the optimal $\alpha_{c}$ is achieved. The traffic load distribution among nodes and links are also studied to give an explanation for the optimal $\alpha$ value.

The paper is organized as follows. In the following section, the traffic model is described in detail, in Sec. III simulation results of traffic dynamics are provided, and Sec. IV gives the conclusion. 


\section{TRAFFIC MODEL}

To generate the underlying network infrastructure, this paper uses a weighted scale-free network model proposed by Wang et al. [24], in which power-law distributions of degrees, weights, and strengths are all in good accordance with real observations of weighted technological networks. In this model, the network is generated with a weight-driven preferential attachment with co-evolution of weights and topology. And the weight-topology co-evolution mimics the traffic interactions of vertices, in contract with previous models where weights are assigned statically [27] or rearranged locally [28]. This model is also different from static complex networks such as those by using fluctuating random graphs [29]. The model rules of [24] can be described as follows. Starting from $m_{0}$ nodes fully connected by links with assigned weight $w_{0}=1$, the system are driven by two mechanics: (1) the strength dynamics: the weight of each link connecting $i$ and $j$ is updated as $w_{i j} \rightarrow w_{i j}+1$, with probability $P_{i j}=W \times p_{i j}=W \times \frac{s_{i} s_{j}}{\sum_{a<b} s_{a} s_{b}}$, where $s_{i}=\sum_{j \in \Gamma_{i}} w_{i j}$ is the strength of node $i$ and $\Gamma_{i}$ is the neighboring set of node $i$; (2) the topological growth: a new node $n$ is added with $m$ links that are randomly attached to a node $i$ according to the strength preferential probability: $\Pi_{n \rightarrow i}=\frac{s_{i}}{\sum_{j} s_{j}}$, where $j$ runs over all existing nodes. Analysis of this model [30] shows that the outcome strength distribution follows a power law $P(s) \sim s^{-\Theta}$ with the exponent $\Theta=2+m /(m+2 W)$. And the exponent $\gamma$ of power-law degree distribution $P(k) \sim k^{-\gamma}$ can be expressed as $\gamma=\phi(\Theta-1)+1$ with $\phi>1$.

Once the network is generated, it remains fixed, and the traffic dynamics is modeled on top of it as follows. At each time step, there are $R$ packets generated homogeneously on the nodes in the system. We treat all the nodes as both hosts and routers and assume that node $i$ can deliver at most $C_{i}=\max \left(\gamma s_{i}, 1\right)$ packets per time step towards their destinations, where $s_{i}$ denotes strength of node $i$. To navigate packets in the system, all the nodes perform a parallel local search among their immediate neighbors, i.e., following a depth $=1$ searching algorithm. If a packet's destination is found within the searched area of node $l$, i.e. the immediate neighbors of $l$, the packet will be delivered from $l$ directly to its target and then removed from the system. Otherwise, the packet will be delivered to a neighboring node $i$ according to the probability:

$$
P_{l \rightarrow i}=\frac{w_{l i}^{\alpha}}{\sum_{j} w_{l j}^{\alpha}},
$$

where $w_{l i}$ is the weight of the link connecting nodes $l$ and $i$, the sum runs over the immediate neighbors of the node $l$, and $\alpha$ is an introduced tunable parameter characterizing the preferential 


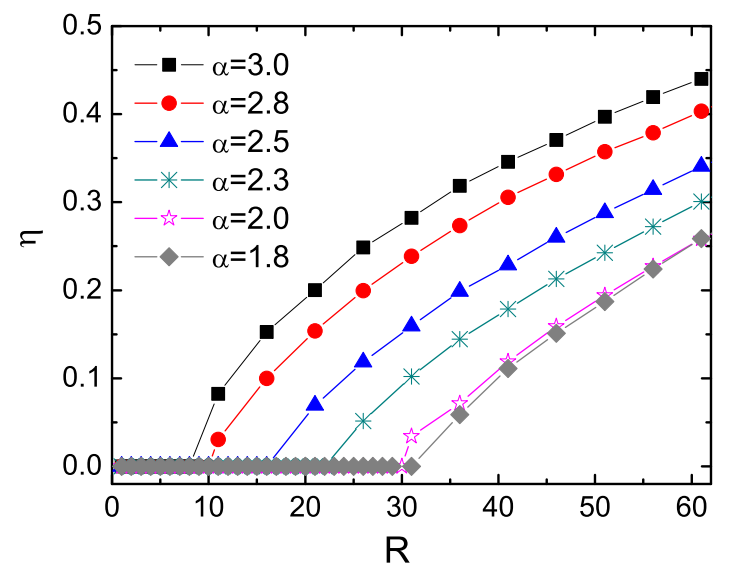

FIG. 1: (Color online) The order parameter $\eta$ versus $R$ for weighted scale-free networks with different routing parameter $\alpha$. Other parameters are networks size $N=1000, m 0=m=5, W=2$ and $\beta=1$.

probability in choosing links to forward packets. Furthermore, the capacity (or bandwidth) of the link connecting nodes $l$ and $i$ is set to $B_{l i}=\max \left(\beta w_{l i}, 1\right)$, i.e., the link can handle at most $B_{l i}$ packets from each end per time step. When the link capacity is reached, the delivery of packets will be delayed and wait for the next time step. During the evolution of the system, the FIFO (first-infirst-out) rule is applied on the nodes. In simulation, we find that the value of $\gamma$ does not affect the qualitative behavior of the system. So we set $\gamma=1$ in the following and mainly discuss the effects of $\alpha$ and $\beta$.

\section{SIMULATION RESULTS}

The network overall capacity is measured by the critical generating rate $R_{c}$ at which a continuous phase transition occurs from free state to congestion. Firstly, we investigate the order parameter [10]:

$$
\eta(R)=\lim _{t \rightarrow \infty} \frac{1}{R} \frac{\left\langle\Delta N_{p}\right\rangle}{\Delta t} .
$$

Here $\Delta N_{p}=N_{p}(t+\Delta t)-N_{p}(t),\langle\ldots\rangle$ denotes taking the average over a time window of width $\Delta t$, and $N_{p}(t)$ is the number of packets in the system at time $t$. As shown in Fig. 1 , when $R<R_{c},\langle\Delta N\rangle=0$ and $\eta(R)=0$, corresponding to the case of free-flow state, in which the numbers of added and removed packets are balanced; while $\eta(R)$ increases suddenly from zero at $R=R_{c}$. Therefore a phase transition occurs at $R=R_{c}$ where congestion emerges and spreads in the system, and packets 
will continuously accumulate in the system. Hence, the system's overall handling and delivering capacity can be measured by the critical value of $R_{c}$. We note that there can be different ways to define the network capacity, but we will mainly consider the critical value of $R_{c}$ in the following discussion.

In Fig.1, one can also see that $\eta(R) \simeq\left|R-R_{c}\right|^{x}$ (when $R \rightarrow R_{c}^{+}$), and the critical exponent $x$ increases with increasing $\alpha$. This indicates that the routing strategy is further away from the optimum, and thus the accumulation rate of packets in the system will increase also.

Our simulations show that when $\beta \rightarrow \infty$, i.e., when the capacity of every link is very large, the maximum network capacity is reached when $\alpha$ is slightly larger than 1.0. Figure 2 and 3 show the overall traffic capacity measured by $R_{c}$ vs $\alpha$ with different $\beta$ values. One can see that both the network capacity and the values of $\alpha_{c}$ remain unchanged when $\beta$ is larger than a threshold $\beta_{c 1} \approx 5$. The maximum capacity always occurs at $\alpha_{c}=1.2$ with $R_{c}^{\max } \approx 313$. This behavior implies that when $\beta>\beta_{c 1}$, the links are operating efficiently under their maximum capacity, and the network capacity is mainly controlled by the node capacity. When $\beta_{c 1}>\beta>\beta_{c 2} \approx 1$, the system's overall capacity decreases rapidly, and the $\alpha_{c}$ value increases with the decrease of $\beta$ (Fig.2). This is because the capacity of some links will be reached from time to time, and thus the delivery of some packets are delayed and accordingly the network capacity decreases. Nevertheless, when $\beta$ becomes smaller than the second threshold $\beta_{c 2} \approx 1, \alpha_{c}$ begins to decrease with the decrease of $\beta$. As we can see in Fig.3, when $\beta=0$, i.e., all links have the same capacity that is equal to one, the system's maximum capacity occurs at $\alpha_{c}=0.2$ with $R_{c}^{\max }=24$.

In addition, simulations also show that $R_{c}$ tends to be a constant with the decrease of $\alpha$. This constant decreases with the decrease of $\beta$ for $\beta_{c 2}<\beta<\beta_{c 1}$. When $\alpha<0$ and $|\alpha|$ is very large, the probability that links with large weight are chosen to deliver packets is very low. This implies that even if we cut off the links with large weight, the network capacity will remain essentially unchanged.

These findings are different from the results obtained by using local routing strategies on unweighted scale-free networks. In [19], Wang et al found that in a typical Barabási-Albert network, the maximum traffic capacity appears at $\alpha_{c}=-1.0$ when the node capacity is set to const. This means to repel the packets from the central nodes and to make them move along the periphery of the network. When considering the heterogeneity of node capacity, they found that $\alpha_{c}=0.0$ when $C=k$. This means that random walk is the best strategy for the packets. In the present paper, different from previous results, it is shown that $\alpha_{c}$ is larger than 1.0. This means that the 


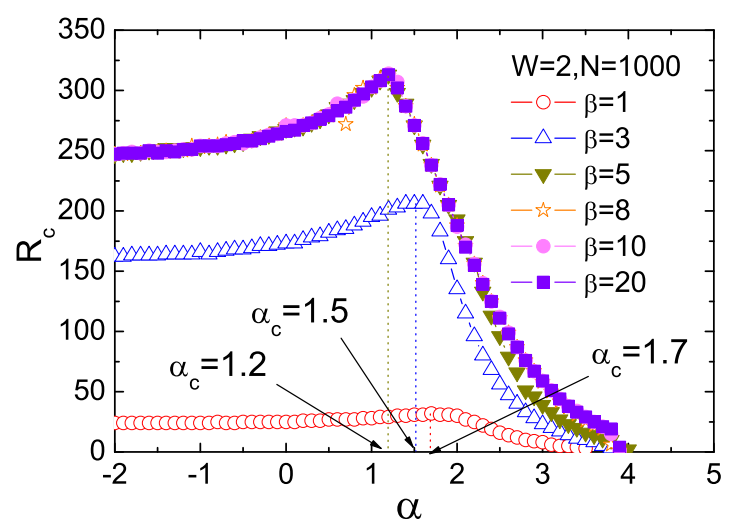

FIG. 2: (Color online) $R_{c}$ vs $\alpha$ with fixed $W=2$ and $\beta \geq 1.0$. The results are obtained by averaging $R_{c}$ over ten network realizations.

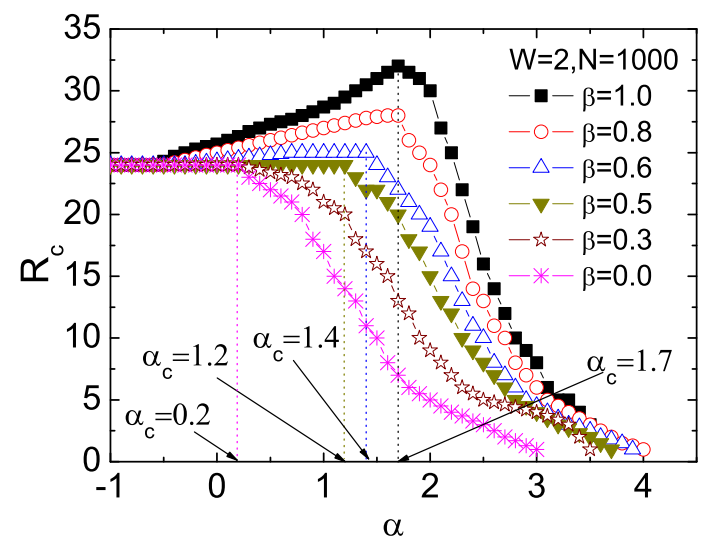

FIG. 3: (Color online) $R_{c}$ vs $\alpha$ with $\beta \leq 1.0$. The results are obtained by averaging $R_{c}$ over ten network realizations.

maximum traffic capacity is achieved by using links with large values of weight. This finding is valuable since most real networks are weighted, and the traffic will probably be affected by the link bandwidth [21].

The analytical estimation of the $R_{c}$ value is very complicated for the weighted traffic system and our routing model. In the following, we give a heuristic explanation for the optimal $\alpha_{c}$ value corresponding to the peak value of $R_{c}$ by investigating the traffic load distribution on the network. In Fig.4, we investigate the average visits per node divided by the node strength which can be used 


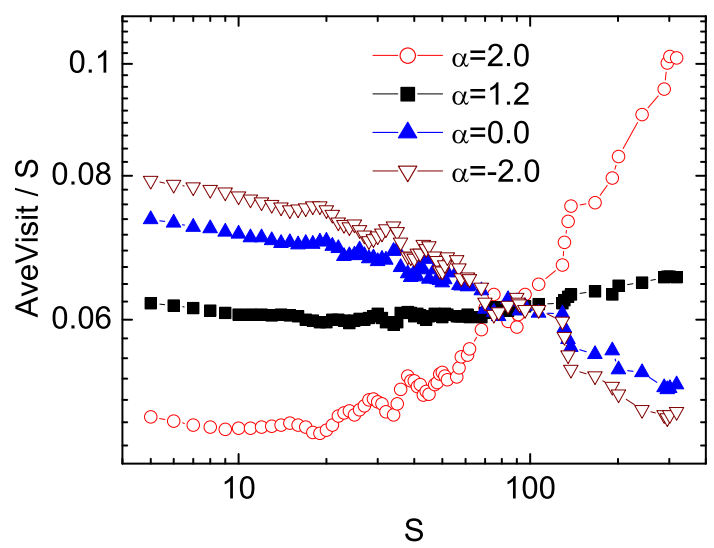

FIG. 4: (Color online) Average visits per node divided by the node strength. The other parameters are $N=1000, m 0=m=5, W=2, R=20$ and $\beta=1$.

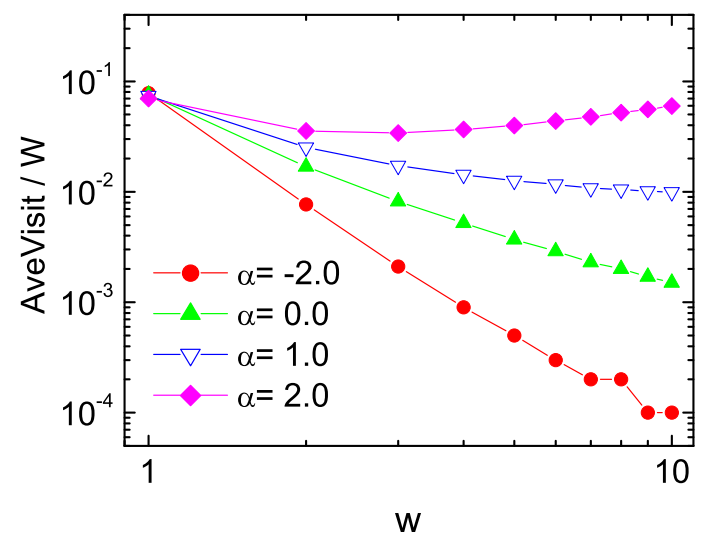

FIG. 5: (Color online) Average visits per link divided by the link weight. The other parameters are $N=$ $1000, m 0=m=5, W=2, R=20$ and $\beta=1$.

to analyze the traffic load distribution among the nodes. When $\alpha<1.2$, the value self-organizes to a power law, which implies that the traffic burden of high-degree nodes is alleviated, while when $\alpha>1.2$, it is an increasing function with respect to $S$, which may lead to the collapse of hubnodes. When $\alpha=1.2$, it essentially remains constant with respect to $S$. At this point, the balance of node capacity with traffic load is achieved for all nodes if the restriction of link bandwidth can be neglected. Since in scale-free networks, the congestion of one node will spread to other nodes 
and trigger the decrement of overall efficiency, this balance ensures that no node will be more easy to jam than others and thus the optimal traffic capacity will be achieved. This conclusion is confirmed by our previous simulation result that the optimal $\alpha_{c}$ value is always located at 1.2 when $\beta \geq 5$.

To explain why $\alpha_{c}>1.2$ for low $\beta$ value, we investigate the average visits per link divided by the link weight which reflects the traffic load distribution among the links. Figure 5 shows the value vs $w$ (link weight) for different $\alpha$ values. One can see that when $\alpha \approx 2.0$, the value is roughly constant for all the link weights. When $\alpha<2.0$, it self-organizes to a power-law, especially when $\alpha$ is smaller than zero. Thus we can see that the capacity of the links will be more fully used when $\alpha$ is set to the value close to 2.0 for the case of $\beta=1$, and therefore the overall capacity of the system will be maximized. We also note that the value remains almost the same for links with weight $w=1$. As in our network model, the weights of most of the links in the system $(\sim 95 \%)$ are equal to one, this behavior explains why the system's capacity will remain constant for negative $\alpha$ values.

Finally, we briefly introduce the effect of link weight growth rate $W$ on the packet traffic capacity. Since $W$ is just a multiplicative factor, the qualitative behavior is not affected by varying $W$. In general, the system's overall capacity will increase with the increase of $W$, but the optimal value of $\alpha_{c}$ remains the same.

When changing the node capacity parameter $\gamma$, it is found that $\alpha_{c}$ depends on $\beta$ in the same way. However, $\beta_{c 1}$ is a monotonically increasing function of $\gamma$, while the value of $\beta_{c 2}$ is independent of $\gamma$. And the maximum value of $R_{c}$ is linearly proportional to $\gamma$.

\section{CONCLUSION}

In conclusion, the traffic dynamics on weighted scale-free networks is studied with a local routing strategy based on link weight information. The simulation yields some results different from previous studies. In general, the overall capacity decreases when the bandwidth parameter of links $(\beta)$ is below a critical value $\beta_{c 1}$. In most cases, the optimal value of local routing parameter $\alpha$ appears at $\alpha_{c}>1.0$, which means to take advantage of links with large bandwidth. And the $\alpha_{c}$ value also depends on $\beta$. We give explanations for the variation of $\alpha_{c}$ by investigating the average visits per node and average visits per link, which are also important for studying the traffic load distribution among the nodes and links. 


\section{Acknowledgement}

This work is funded by National Basic Research Program of China (No.2006CB705500), the NNSFC under Key Project No.10532060, Project Nos.70601026, 10672160, the CAS President Foundation, and the China Postdoctoral Science Foundation (No. 20060390179). Y.-H. Wu acknowledges the support of Australian Research Council through a Discovery Project Grant.

[1] D.J. Watts, S.H. Strogatz, Nature 393, 440 (1998).

[2] A.L. Barabási, R. Albert, Science 286, 509 (1999).

[3] M.E.J. Newman, SIAM Rev. 45, 167 (2003).

[4] S. Boccaletti, V. Latora, Y. Moreno et al, Phys. Rep. 424, 175 (2006).

[5] J.J. Wu, H.J. Sun, Z.Y. Gao, Physica A 386(1), 407(2007).

[6] M. Li, F. Liu, F.Y. Ren, Phys. Rev. E 75, 066115 (2007).

[7] X.J. Xu, X. Zhang, J.F.F. Mendes, Phys. Rev. E 76(5), 056109 (2007).

[8] X.J. Xu, Z.X. Wu, G.R. Chen, Physica A 377, 125 (2007).

[9] H.J. Yang, F.C. Zhao, B.H. Wang, Physica A 364, 544 (2006).

[10] A. Arenas, A. Díaz-Guilerà, R. Guimerà, Phys. Rev. Lett. 86, 3196 (2001).

[11] R. Guimerà, A. Díaz-Guilera, F. Vega-Redondo et al, Phys. Rev. Lett. 89, 248701 (2002).

[12] B. Tadić, S. Thurner, G.J. Rodgers, Phys. Rev. E 69, 036102 (2004).

[13] B. Tadić, G.J. Rodgers, S. Thurner, Int. J. Bifurcation and Chaos 7, 2363 (2007).

[14] B. Tadić, S. Thurner, Physica A 346, 183(2005).

[15] J.D. Noh, H. Rieger, Phys. Rev. Lett. 92, 118701 (2004).

[16] L. Zhao, K. Park, Y.C. Lai, Phys. Rev. E 70, 035101(R) (2004).

[17] G. Yan, T. Zhou, B. Hu et al, Phys. Rev. E 73, 046108 (2006).

[18] A.P.S. de Moura, Phys. Rev. E 71, 066114 (2005).

[19] W.X. Wang, B.H. Wang, C.Y. Yin et al. Phys. Rev. E. 73, 026111 (2006).

[20] M.B. Hu, W.X. Wang, R. Jiang et al. Phys. Rev. E 75, 036102 (2007).

[21] M.B. Hu, W.X. Wang, R. Jiang et al. Europhys. Lett. 79, 14003 (2007).

[22] C.Y. Yin, B.H. Wang, W.X. Wang et al. Eur. Phys. J. B 49, 205 (2006).

[23] W.X. Wang, C.Y. Yin, G. Yan et al. Phys. Rev. E 74, 016101 (2006). 
[24] W.X. Wang, B.H. Wang, B. Hu et al, Phys. Rev. Lett. 94, 188702 (2005).

[25] A. Barrat, M. Barthélemy et al. Proc. Natl. Acad. Sci. U.S.A. 101,3747(2004).

[26] P.J. Macdonald, E. Almaas, A.L. Barabási, Europhys. Lett. 72, 308 (2005).

[27] S.N. Dorogovtsev, J.F.F. Mendes, Europhys. Lett. 52, 33 (2000).

[28] A. Barrat, M. Barthélemy, A. Vespignani, Phys. Rev. Lett. 92,228701(2004).

[29] S. Abe, S. Thurner, Phys. Rev. E, 72, 036102 (2005).

[30] Y.B. Xie, W.X. Wang, B.H. Wang, Phys. Rev. E 75, 026111 (2007).

[31] A.E. Motter, C.S. Zhou, J. Kurths, Phys. Rev. E, 71, 016116(2005).

[32] C.S. Zhou, A.E. Motter, J. Kurths, Phys. Rev. Lett. 96, 034101(2006).

[33] Z.Z. Zhang, S.G. Zhou, L.C. Chen et al. Euro. Phys. J. B 59, 99 (2007). 